\title{
Polyrectangular theorem and independence of uncertain vectors
}

\author{
Baoding Liu
}

Correspondence:

liu@tsinghua.edu.cn

Department of Mathematical

Sciences, Tsinghua University,

Beijing 100084, China

\begin{abstract}
Uncertainty theory is a branch of mathematics for modeling human uncertainty. This paper gives a concept of polyrectangle in product uncertainty space and proves a polyrectangular theorem. This paper also proposes a concept of independence of uncertain vectors and discusses some mathematical properties of it. Finally, this paper presents a concept of normal uncertain vector with multivariate normal distribution.
\end{abstract}

Keywords: Uncertainty theory; Uncertain measure; Uncertain vector; Polyrectangular theorem

\section{Background}

Real decisions are usually made in the state of indeterminacy. For modeling indeterminacy, there exist two mathematical systems, one is probability theory [1], and the other is uncertainty theory [2]. Probability is interpreted as frequency, while uncertainty is interpreted as personal belief degree [3].

Uncertainty theory was founded by Liu [2] in 2007 and perfected by Liu [4] in 2009 with the fundamental concept of uncertain measure. After that, Gao [5], Zhang [6], and Peng and Iwamura [7] also discussed some mathematical properties of uncertain measure. In many applications of uncertainty theory, we have to deal with uncertain events which are not necessarily independent. In order to study the dependence of uncertain events, Liu [2] defined a conditional uncertain measure of an event after it has been learned that some other event has occurred. Since then, the tool of uncertain measure was well developed and became a rigorous footstone of uncertainty theory.

As another fundamental concept in uncertainty theory, the uncertain variable was presented by Liu [2] in 2007. In order to describe uncertain variable, Liu [2] also introduced the concept of uncertainty distribution. Later, Peng and Iwamura [8] proved a sufficient and necessary condition for uncertainty distribution. In addition, Liu [9] proposed the concept of inverse uncertainty distribution, and Liu [10] verified a sufficient and necessary condition for it. Furthermore, a measure inversion theorem was given by Liu [9] that may yield uncertain measures of some special events from the uncertainty distribution of the corresponding uncertain variable.

Following the independence of uncertain variables proposed by Liu [4], the operational law was given by Liu [9] for calculating the uncertainty distribution and inverse uncertainty distribution of strictly monotone function of independent uncertain variables.

(C) 2013 Liu; licensee Springer. This is an Open Access article distributed under the terms of the Creative Commons Attribution License (http://creativecommons.org/licenses/by/2.0), which permits unrestricted use, distribution, and reproduction in any medium, provided the original work is properly cited. 
In order to rank the uncertain variables, Liu [2] proposed the concept of expected value operator. In addition, the linearity of expected value operator was verified by Liu [9]. As an important contribution, Liu and Ha [11] derived a useful formula for calculating the expected values of monotone functions of uncertain variables. Based on the expected value operator, Liu [2] presented the concepts of variance and moments of uncertain variables. Furthermore, some variance formulas were given by Yao [12].

This paper will give a concept of polyrectangle in product uncertainty space and prove a polyrectangular theorem. This paper will also propose a concept of independence of uncertain vectors and discuss some mathematical properties of it. Finally, this paper will present a concept of normal uncertain vector with multivariate normal distribution.

\section{Uncertainty theory}

Let $\Gamma$ be a nonempty set, and $\mathcal{L}$ a $\sigma$-algebra over $\Gamma$. Each element $\Lambda$ in $\mathcal{L}$ is called an event. Liu [2] defined an uncertain measure by the following axioms:

Axiom 1. Normality axiom: $\mathcal{M}\{\Gamma\}=1$ for the universal set $\Gamma$;

Axiom 2. Duality axiom: $\mathcal{M}\{\Lambda\}+\mathcal{M}\left\{\Lambda^{c}\right\}=1$ for any event $\Lambda$;

Axiom 3. Subadditivity axiom: For every countable sequence of events $\Lambda_{1}, \Lambda_{2}, \cdots$, we have

$$
\mathcal{M}\left\{\bigcup_{i=1}^{\infty} \Lambda_{i}\right\} \leq \sum_{i=1}^{\infty} \mathcal{M}\left\{\Lambda_{i}\right\} .
$$

The triplet $(\Gamma, \mathcal{L}, \mathcal{M})$ is called an uncertainty space. Furthermore, Liu [4] defined a product uncertain measure by the fourth axiom:

Axiom 4. Product axiom: Let $\left(\Gamma_{k}, \mathcal{L}_{k}, \mathcal{M}_{k}\right)$ be uncertainty spaces for $k=1,2, \ldots$. The product uncertain measure $\mathcal{M}$ is an uncertain measure satisfying

$$
\mathcal{M}\left\{\prod_{k=1}^{\infty} \Lambda_{k}\right\}=\bigwedge_{k=1}^{\infty} \mathcal{M}_{k}\left\{\Lambda_{k}\right\},
$$

where $\Lambda_{k}$ are arbitrarily chosen events from $\mathcal{L}_{k}$ for $k=1,2, \cdots$, respectively.

An uncertain variable is defined by Liu [2] as a function $\xi$ from an uncertainty space $(\Gamma, \mathcal{L}, \mathcal{M})$ to the set of real numbers, such that $\{\xi \in B\}$ is an event for any Borel set $B$. In order to describe an uncertain variable in practice, the concept of uncertainty distribution is defined by Liu [2] as

$$
\Phi(x)=\mathcal{M}\{\xi \leq x\}, \quad \forall x \in \Re .
$$

Peng and Iwamura [8] verified that a function $\Phi: \Re \rightarrow[0,1]$ is an uncertainty distribution if and only if it is a monotone increasing function except $\Phi(x) \equiv 0$ and $\Phi(x) \equiv 1$. An uncertainty distribution $\Phi(x)$ is said to be regular if it is a continuous and strictly increasing function with respect to $x$ at which $0<\Phi(x)<1$, and

$$
\lim _{x \rightarrow-\infty} \Phi(x)=0, \quad \lim _{x \rightarrow+\infty} \Phi(x)=1 .
$$


Let $\xi$ be an uncertain variable with regular uncertainty distribution $\Phi(x)$. Then, the inverse function $\Phi^{-1}(\alpha)$ is called the inverse uncertainty distribution of $\xi$ [9]. It is also verified by Liu [10] that a function $\Phi^{-1}(\alpha):(0,1) \rightarrow \Re$ is an inverse uncertainty distribution if and only if it is a continuous and strictly increasing function with respect to $\alpha$.

The expected value of an uncertain variable $\xi$ is defined by Liu [2] as the following form

$$
E[\xi]=\int_{0}^{+\infty} \mathcal{M}\{\xi \geq x\} \mathrm{d} x-\int_{-\infty}^{0} \mathcal{M}\{\xi \leq x\} \mathrm{d} x,
$$

provided that at least one of the two integrals is finite. If $\xi$ has an uncertainty distribution $\Phi$, then the expected value may be calculated by

$$
E[\xi]=\int_{0}^{+\infty}(1-\Phi(x)) \mathrm{d} x-\int_{-\infty}^{0} \Phi(x) \mathrm{d} x .
$$

Let $\xi$ be an uncertain variable with finite expected value $e$. Then, the variance of $\xi$ is defined by Liu [2] as

$$
V[\xi]=E\left[(\xi-e)^{2}\right] .
$$

If $\xi$ has an uncertainty distribution $\Phi$, then

$$
V[\xi]=\int_{0}^{+\infty}(1-\Phi(e+\sqrt{x})+\Phi(e-\sqrt{x})) \mathrm{d} x .
$$

Independence is an extremely important concept in uncertainty theory. The uncertain variables $\xi_{1}, \xi_{2}, \cdots, \xi_{n}$ are said to be independent [4] if

$$
\mathcal{M}\left\{\bigcap_{i=1}^{n}\left(\xi_{i} \in B_{i}\right)\right\}=\bigwedge_{i=1}^{n} \mathcal{M}\left\{\xi_{i} \in B_{i}\right\}
$$

for any Borel sets $B_{1}, B_{2}, \cdots, B_{n}$ of real numbers. Equivalently, those uncertain variables are independent if and only if

$$
\mathcal{M}\left\{\bigcup_{i=1}^{n}\left(\xi_{i} \in B_{i}\right)\right\}=\bigvee_{i=1}^{n} \mathcal{M}\left\{\xi_{i} \in B_{i}\right\}
$$

Let $\xi_{1}, \xi_{2}, \cdots, \xi_{n}$ be independent uncertain variables with uncertainty distributions $\Phi_{1}, \Phi_{2}, \cdots, \Phi_{n}$, respectively. Assume that the function $f\left(x_{1}, x_{2}, \cdots, x_{n}\right)$ is strictly increasing with respect to $x_{1}, x_{2}, \cdots, x_{m}$ and strictly decreasing with respect to $x_{m+1}, x_{m+2}, \cdots, x_{n}$. Liu [9] showed that $\xi=f\left(\xi_{1}, \xi_{2}, \cdots, \xi_{n}\right)$ has an inverse uncertainty distribution

$$
\Psi^{-1}(\alpha)=f\left(\Phi_{1}^{-1}(\alpha), \cdots, \Phi_{m}^{-1}(\alpha), \Phi_{m+1}^{-1}(1-\alpha), \cdots, \Phi_{n}^{-1}(1-\alpha)\right)
$$

In addition, Liu and $\mathrm{Ha}[11]$ proved that the uncertain variable $\xi$ has an expected value

$$
E[\xi]=\int_{0}^{1} f\left(\Phi_{1}^{-1}(\alpha), \cdots, \Phi_{m}^{-1}(\alpha), \Phi_{m+1}^{-1}(1-\alpha), \cdots, \Phi_{n}^{-1}(1-\alpha)\right) \mathrm{d} \alpha .
$$

Furthermore, Yao [12] proved that $\xi$ has a variance

$$
V[\xi]=\int_{0}^{1}\left(f\left(\Phi_{1}^{-1}(\alpha), \cdots, \Phi_{m}^{-1}(\alpha), \Phi_{m+1}^{-1}(1-\alpha), \cdots, \Phi_{n}^{-1}(1-\alpha)\right)-E[\xi]\right)^{2} \mathrm{~d} \alpha .
$$




\section{Independence of events}

Consider an uncertainty space $(\Gamma, \mathcal{L}, \mathcal{M})$. The events $\Lambda_{1}, \Lambda_{2}, \cdots, \Lambda_{n}$ are said to be independent [9] if

$$
\mathcal{M}\left\{\bigcap_{i=1}^{n} \Lambda_{i}^{*}\right\}=\bigwedge_{i=1}^{n} \mathcal{M}\left\{\Lambda_{i}^{*}\right\},
$$

where $\Lambda_{i}^{*}$ are arbitrarily chosen from $\left\{\Lambda_{i}, \Lambda_{i}^{c}\right\}, i=1,2, \cdots, n$, respectively.

Note that (13) represents $2^{n}$ equations. For example, when $n=2$, the four equations are

$$
\begin{aligned}
& \mathcal{M}\left\{\Lambda_{1} \cap \Lambda_{2}\right\}=\mathcal{M}\left\{\Lambda_{1}\right\} \wedge \mathcal{M}\left\{\left\{\Lambda_{2}\right\}\right. \\
& \mathcal{M}\left\{\Lambda_{1}^{c} \cap \Lambda_{2}\right\}=\mathcal{M}\left\{\Lambda_{1}^{c}\right\} \wedge \mathcal{M}\left\{\left\{\Lambda_{2}\right\}\right. \\
& \mathcal{M}\left\{\Lambda_{1} \cap \Lambda_{2}^{c}\right\}=\mathcal{M}\left\{\Lambda_{1}\right\} \wedge \mathcal{M}\left\{\left\{\Lambda_{2}^{c}\right\}\right. \\
& \mathcal{M}\left\{\Lambda_{1}^{c} \cap \Lambda_{2}^{c}\right\}=\mathcal{M}\left\{\Lambda_{1}^{c}\right\} \wedge \mathcal{M}\left\{\left\{\Lambda_{2}^{c}\right\}\right.
\end{aligned}
$$

Theorem 1. Let $\left(\Gamma_{i}, \mathcal{L}_{i}, \mathcal{M}_{i}\right)$ be uncertainty spaces and $\Lambda_{i} \in \mathcal{L}_{i}$ for $i=1,2, \cdots, n$. Then, the events

$$
\Gamma_{1} \times \cdots \times \Gamma_{i-1} \times \Lambda_{i} \times \Gamma_{i+1} \times \cdots \times \Gamma_{n}, \quad i=1,2, \cdots, n
$$

are always independent in the product uncertainty space. That is, the events

$$
\Lambda_{1}, \Lambda_{2}, \cdots, \Lambda_{n}
$$

are always independent if they are from different uncertainty spaces.

Proof. For simplicity, we only prove the case of $n=2$. It follows from the product axiom that the product uncertain measure of the intersection is

$$
\mathcal{M}\left\{\left(\Lambda_{1} \times \Gamma_{2}\right) \cap\left(\Gamma_{1} \times \Lambda_{2}\right)\right\}=\mathcal{M}\left\{\Lambda_{1} \times \Lambda_{2}\right\}=\mathcal{M}_{1}\left\{\Lambda_{1}\right\} \wedge \mathcal{M}_{2}\left\{\Lambda_{2}\right\}
$$

Using $\mathcal{M}\left\{\Lambda_{1} \times \Gamma_{2}\right\}=\mathcal{M}_{1}\left\{\Lambda_{1}\right\}$ and $\mathcal{M}\left\{\Gamma_{1} \times \Lambda_{2}\right\}=\mathcal{M}_{2}\left\{\Lambda_{2}\right\}$, we obtain

$$
\mathcal{M}\left\{\left(\Lambda_{1} \times \Gamma_{2}\right) \cap\left(\Gamma_{1} \times \Lambda_{2}\right)\right\}=\mathcal{M}\left\{\Lambda_{1} \times \Gamma_{2}\right\} \wedge \mathcal{M}\left\{\Gamma_{1} \times \Lambda_{2}\right\} .
$$

Similarly, we may prove that

$$
\begin{aligned}
\mathcal{M}\left\{\left(\Lambda_{1} \times \Gamma_{2}\right)^{c} \cap\left(\Gamma_{1} \times \Lambda_{2}\right)\right\} & =\mathcal{H}\left\{\left(\Lambda_{1} \times \Gamma_{2}\right)^{c}\right\} \wedge \mathcal{N}\left\{\Gamma_{1} \times \Lambda_{2}\right\}, \\
\mathcal{M}\left\{\left(\Lambda_{1} \times \Gamma_{2}\right) \cap\left(\Gamma_{1} \times \Lambda_{2}\right)^{c}\right\} & =\mathcal{H}\left\{\Lambda_{1} \times \Gamma_{2}\right\} \wedge \mathcal{M}\left\{\left(\Gamma_{1} \times \Lambda_{2}\right)^{c}\right\}, \\
\mathcal{H}\left\{\left(\Lambda_{1} \times \Gamma_{2}\right)^{c} \cap\left(\Gamma_{1} \times \Lambda_{2}\right)^{c}\right\} & =\mathcal{N}\left\{\left(\Lambda_{1} \times \Gamma_{2}\right)^{c}\right\} \wedge \mathcal{M}\left\{\left(\Gamma_{1} \times \Lambda_{2}\right)^{c}\right\} .
\end{aligned}
$$

Thus, $\Lambda_{1} \times \Gamma_{2}$ and $\Gamma_{1} \times \Lambda_{2}$ are independent events. Furthermore, since $\Lambda_{1}$ and $\Lambda_{2}$ are understood as $\Lambda_{1} \times \Gamma_{2}$ and $\Gamma_{1} \times \Lambda_{2}$ in the product uncertainty space, respectively, the two events $\Lambda_{1}$ and $\Lambda_{2}$ are also independent.

\section{Polyrectangular theorem}

Let $\left(\Gamma_{1}, \mathcal{L}_{1}, \mathcal{M}_{1}\right)$ and $\left(\Gamma_{2}, \mathcal{L}_{2}, \mathcal{M}_{2}\right)$ be two uncertainty spaces, $\Lambda_{1} \in \mathcal{L}_{1}$ and $\Lambda_{2} \in \mathcal{L}_{2}$. It follows from the product axiom that the rectangle $\Lambda_{1} \times \Lambda_{2}$ has an uncertain measure

$$
\mathcal{N}\left\{\Lambda_{1} \times \Lambda_{2}\right\}=\mathcal{M}_{1}\left\{\Lambda_{1}\right\} \wedge \mathcal{M}_{2}\left\{\Lambda_{2}\right\}
$$

This section will extend this result to a more general case. 
Definition 1. Let $\left(\Gamma_{1}, \mathcal{L}_{1}, \mathcal{M}_{1}\right)$ and $\left(\Gamma_{2}, \mathcal{L}_{2}, \mathcal{M}_{2}\right)$ be two uncertainty spaces. A set on $\Gamma_{1} \times \Gamma_{2}$ is called a polyrectangle if it has the form

$$
\Lambda=\bigcup_{i=1}^{m}\left(\Lambda_{1 i} \times \Lambda_{2 i}\right)
$$

where $\Lambda_{1 i} \in \mathcal{L}_{1}$, and $\Lambda_{2 i} \in \mathcal{L}_{2}$ for $i=1,2, \cdots, m$, and

$$
\begin{aligned}
& \Lambda_{11} \subset \Lambda_{12} \subset \cdots \subset \Lambda_{1 m}, \\
& \Lambda_{21} \supset \Lambda_{22} \supset \cdots \supset \Lambda_{2 m} .
\end{aligned}
$$

Theorem 2. Polyrectangular Theorem: Let $\left(\Gamma_{1}, \mathcal{L}_{1}, \mathcal{M}_{1}\right)$ and $\left(\Gamma_{2}, \mathcal{L}_{2}, \mathcal{M}_{2}\right)$ be two uncertainty spaces. Then, the polyrectangle

$$
\Lambda=\bigcup_{i=1}^{m}\left(\Lambda_{1 i} \times \Lambda_{2 i}\right)
$$

on the product uncertainty space $\left(\Gamma_{1}, \mathcal{L}_{1}, \mathcal{M}_{1}\right) \times\left(\Gamma_{2}, \mathcal{L}_{2}, \mathcal{M}_{2}\right)$ has an uncertain measure

$$
\mathcal{M}\{\Lambda\}=\bigvee_{i=1}^{m} \mathcal{M}_{1}\left\{\Lambda_{1 i}\right\} \wedge \mathcal{M}_{2}\left\{\Lambda_{2 i}\right\}
$$

Proof. It is clear that the maximum rectangle in the polyrectangle $\Lambda$ is one of $\Lambda_{1 i} \times \Lambda_{2 i}$, $i=1,2, \cdots, n$. Denote the maximum rectangle by $\Lambda_{1 k} \times \Lambda_{2 k}$.

Case I: If

$$
\mathcal{M}\left\{\Lambda_{1 k} \times \Lambda_{2 k}\right\}=\mathcal{M}_{1}\left\{\Lambda_{1 k}\right\}
$$

then, the maximum rectangle in $\Lambda^{c}$ is $\Lambda_{1 k}^{c} \times \Lambda_{2, k+1}^{c}$, and

$$
\mathcal{M}\left\{\Lambda_{1 k}^{c} \times \Lambda_{2, k+1}^{c}\right\}=\mathcal{M}_{1}\left\{\Lambda_{1 k}^{c}\right\}=1-\mathcal{M}_{1}\left\{\Lambda_{1 k}\right\} .
$$

Thus,

$$
\mathcal{M}\left\{\Lambda_{1 k} \times \Lambda_{2 k}\right\}+\mathcal{M}\left\{\Lambda_{1 k}^{c} \times \Lambda_{2, k+1}^{c}\right\}=1 .
$$

Case II: If

$$
\mathcal{M}\left\{\Lambda_{1 k} \times \Lambda_{2 k}\right\}=\mathcal{M}_{2}\left\{\Lambda_{2 k}\right\},
$$

then the maximum rectangle in $\Lambda^{c}$ is $\Lambda_{1, k-1}^{c} \times \Lambda_{2 k}^{c}$, and

$$
\mathcal{M}\left\{\Lambda_{1, k-1}^{c} \times \Lambda_{2 k}^{c}\right\}=\mathcal{M}_{2}\left\{\Lambda_{2 k}^{c}\right\}=1-\mathcal{M}_{2}\left\{\Lambda_{2 k}\right\} .
$$

Thus,

$$
\mathcal{M}\left\{\Lambda_{1 k} \times \Lambda_{2 k}\right\}+\mathcal{M}\left\{\Lambda_{1, k-1}^{c} \times \Lambda_{2 k}^{c}\right\}=1 .
$$

No matter what case happens, the sum of the uncertain measures of the maximum rectangles in $\Lambda$ and $\Lambda^{c}$ is always 1 . It follows from the product axiom that (21) holds.

\section{Independence of uncertain vectors}

A $k$-dimensional uncertain vector is a function $\xi$ from an uncertainty space $(\Gamma, \mathcal{L}, \mathcal{M})$ to the set of $k$-dimensional real vectors such that $\{\xi \in B\}$ is an event for any $k$-dimensional 
Borel set $B$ [2]. It has been proved that $\left(\xi_{1}, \xi_{2}, \cdots, \xi_{k}\right)$ is an uncertain vector if and only if $\xi_{1}, \xi_{2}, \cdots, \xi_{k}$ are uncertain variables. The joint uncertainty distribution of an uncertain vector $\left(\xi_{1}, \xi_{2}, \cdots, \xi_{k}\right)$ is defined by Liu [2] as

$$
\Phi\left(x_{1}, x_{2}, \cdots, x_{k}\right)=\mathcal{M}\left\{\xi_{1} \leq x_{1}, \xi_{2} \leq x_{2}, \cdots, \xi_{k} \leq x_{k}\right\}
$$

for any real numbers $x_{1}, x_{2}, \cdots, x_{k}$.

Theorem 3. Let $\xi_{1}, \xi_{2}, \cdots, \xi_{k}$ be independent uncertain variables with uncertainty distributions $\Phi_{1}, \Phi_{2}, \cdots, \Phi_{k}$, respectively. Then, the uncertain vector $\left(\xi_{1}, \xi_{2}, \cdots, \xi_{k}\right)$ has a joint uncertainty distribution

$$
\Phi\left(x_{1}, x_{2}, \cdots, x_{k}\right)=\Phi_{1}\left(x_{1}\right) \wedge \Phi_{2}\left(x_{2}\right) \wedge \cdots \wedge \Phi_{k}\left(x_{k}\right)
$$

for any real numbers $x_{1}, x_{2}, \cdots, x_{k}$.

Proof. Since $\xi_{1}, \xi_{2}, \cdots, \xi_{k}$ are independent uncertain variables, we have

$$
\Phi\left(x_{1}, x_{2}, \cdots, x_{k}\right)=\mathcal{M}\left\{\bigcap_{i=1}^{k}\left(\xi_{i} \leq x_{i}\right)\right\}=\bigwedge_{i=1}^{k} \mathcal{M}\left\{\xi_{i} \leq x_{i}\right\}=\bigwedge_{i=1}^{k} \Phi_{i}\left(x_{i}\right)
$$

for any real numbers $x_{1}, x_{2}, \cdots, x_{k}$. The theorem is proved.

Definition 2. The k-dimensional uncertain vectors $\xi_{1}, \xi_{2}, \cdots, \xi_{n}$ are said to be independent iffor any $k$-dimensional Borel sets $B_{1}, B_{2}, \cdots, B_{n}$, we have

$$
\mathcal{M}\left\{\bigcap_{i=1}^{n}\left(\boldsymbol{\xi}_{i} \in B_{i}\right)\right\}=\bigwedge_{i=1}^{n} \mathcal{M}\left\{\boldsymbol{\xi}_{i} \in B_{i}\right\}
$$

Theorem 4. The $k$-dimensional uncertain vectors $\boldsymbol{\xi}_{1}, \boldsymbol{\xi}_{2}, \cdots, \boldsymbol{\xi}_{n}$ are independent if and only if

$$
\mathcal{M}\left\{\bigcup_{i=1}^{n}\left(\xi_{i} \in B_{i}\right)\right\}=\bigvee_{i=1}^{n} \mathcal{M}\left\{\xi_{i} \in B_{i}\right\}
$$

for any $k$-dimensional Borel sets $B_{1}, B_{2}, \cdots, B_{n}$.

Proof. It follows from the duality of uncertain measure that $\xi_{1}, \xi_{2}, \cdots, \xi_{n}$ are independent if and only if

$$
\begin{aligned}
& \mathcal{M}\left\{\bigcup_{i=1}^{n}\left(\xi_{i} \in B_{i}\right)\right\}=1-\mathcal{M}\left\{\bigcap_{i=1}^{n}\left(\xi_{i} \in B_{i}^{c}\right)\right\} \\
& =1-\bigwedge_{i=1}^{n} \mathcal{M}\left\{\xi_{i} \in B_{i}^{c}\right\}=\bigvee_{i=1}^{n} \mathcal{M}\left\{\xi_{i} \in B_{i}\right\} .
\end{aligned}
$$

The theorem is thus proved.

Theorem 5. Let $\boldsymbol{\xi}_{1}, \boldsymbol{\xi}_{2}, \cdots, \boldsymbol{\xi}_{n}$ be independent uncertain vectors, and $f_{1}, f_{2}, \cdots, f_{n}$ vectorvalued measurable functions. Then, $f_{1}\left(\xi_{1}\right), f_{2}\left(\xi_{2}\right), \cdots, f_{n}\left(\xi_{n}\right)$ are independent uncertain vectors. 
Proof. For any Borel sets $B_{1}, B_{2}, \cdots, B_{n}$, it follows from the definition of independence that

$$
\begin{aligned}
& \mathcal{M}\left\{\bigcap_{i=1}^{n}\left(f_{i}\left(\xi_{i}\right) \in B_{i}\right)\right\}=\mathcal{M}\left\{\bigcap_{i=1}^{n}\left(\xi_{i} \in f_{i}^{-1}\left(B_{i}\right)\right)\right\} \\
& =\bigwedge_{i=1}^{n} \mathcal{M}\left\{\xi_{i} \in f_{i}^{-1}\left(B_{i}\right)\right\}=\bigwedge_{i=1}^{n} \mathcal{M}\left\{f_{i}\left(\xi_{i}\right) \in B_{i}\right\} .
\end{aligned}
$$

Thus, $f_{1}\left(\boldsymbol{\xi}_{1}\right), f_{2}\left(\boldsymbol{\xi}_{2}\right), \cdots, f_{n}\left(\boldsymbol{\xi}_{n}\right)$ are independent uncertain variables.

\section{Multivariate normal distribution}

An uncertain variable $\xi$ is called normal if it has a normal uncertainty distribution

$$
\Phi(x)=\left(1+\exp \left(\frac{\pi(e-x)}{\sqrt{3} \sigma}\right)\right)^{-1}, \quad x \in \Re
$$

denoted by $\mathcal{N}(e, \sigma)$, where $e$ is the expected value, and $\sigma>0$ is the standard variance.

Definition 3. Let $\tau_{1}, \tau_{2}, \cdots, \tau_{m}$ be independent normal uncertain variables with expected value 0 and variance 1 . Then, the uncertain vector

$$
\boldsymbol{\tau}=\left(\tau_{1}, \tau_{2}, \cdots, \tau_{m}\right)
$$

is said to have a multivariate standard normal distribution.

It is easy to verify that a standard normal uncertain vector $\left(\tau_{1}, \tau_{2}, \cdots, \tau_{m}\right)$ has a joint uncertainty distribution

$$
\Phi\left(x_{1}, x_{2}, \cdots, x_{m}\right)=\left(1+\exp \left(-\frac{\pi\left(x_{1} \wedge x_{2} \wedge \cdots \wedge x_{m}\right)}{\sqrt{3}}\right)\right)^{-1}
$$

for any real numbers $x_{1}, x_{2}, \cdots, x_{m}$. It is also easy to show that

$$
\begin{gathered}
\lim _{x_{i} \rightarrow-\infty} \Phi\left(x_{1}, x_{2}, \cdots, x_{m}\right)=0, \text { for each } i, \\
\lim _{\left(x_{1}, x_{2}, \cdots, x_{m}\right) \rightarrow+\infty} \Phi\left(x_{1}, x_{2}, \cdots, x_{m}\right)=1 .
\end{gathered}
$$

Furthermore, the limit

$$
\lim _{\left(x_{1}, \cdots, x_{i-1}, x_{i+1}, \cdots, x_{m}\right) \rightarrow+\infty} \Phi\left(x_{1}, x_{2}, \cdots, x_{m}\right)
$$

is a standard normal distribution with respect to $x_{i}$.

Definition 4. Let $\left(\tau_{1}, \tau_{2}, \cdots, \tau_{m}\right)$ be a standard normal uncertain vector, and let $e_{i}, \sigma_{i j}$, $i=1,2, \cdots, k, j=1,2, \cdots, m$ be real numbers. Define

$$
\xi_{i}=e_{i}+\sum_{j=1}^{m} \sigma_{i j} \tau_{j}
$$

for $i=1,2, \cdots, k$. Then, the uncertain vector $\left(\xi_{1}, \xi_{2}, \cdots, \xi_{k}\right)$ is said to have a multivariate normal distribution.

That is, an uncertain vector $\xi$ has a multivariate normal distribution if it can be represented in the form

$$
\xi=e+\sigma \tau
$$


for some real vector $\boldsymbol{e}$ and some real matrix $\boldsymbol{\sigma}$, where $\boldsymbol{\tau}$ is a standard normal uncertain vector. Note that $\boldsymbol{\xi}, \boldsymbol{e}$ and $\boldsymbol{\tau}$ are understood as column vectors. Please also note that for every index $i$, the component $\xi_{i}$ is a normal uncertain variable with expected value $e_{i}$ and standard variance

$$
\sum_{j=1}^{m}\left|\sigma_{i j}\right| \text {. }
$$

Theorem 6. Assume $\xi$ is a normal uncertain vector, $c$ is a real vector, and $D$ is a real matrix. Then,

$$
\eta=c+D \xi
$$

is another normal uncertain vector.

Proof. Since $\xi$ is a normal uncertain vector, there exist a standard normal uncertain vector $\tau$, a real vector $\boldsymbol{e}$, and a real matrix $\sigma$, such that $\xi=\boldsymbol{e}+\boldsymbol{\sigma} \tau$. It follows that

$$
\boldsymbol{\eta}=c+D \xi=c+D(\boldsymbol{e}+\boldsymbol{\sigma} \boldsymbol{\tau})=(c+D \boldsymbol{e})+(D \boldsymbol{\sigma}) \boldsymbol{\tau} .
$$

Hence, $\boldsymbol{\eta}$ is a normal uncertain vector.

\section{Conclusions}

This paper gave a concept of polyrectangle in product uncertainty space and proved a polyrectangular theorem. This paper also proposed a concept of independence of uncertain vectors and discussed some mathematical properties of it. Finally, this paper presented a concept of normal uncertain vector.

\section{Acknowledgements}

This work was supported by the National Natural Science Foundation of China (grants no.91224008 and no.61273044).

Received: 13 October 2013 Accepted: 24 October 2013

Published: 1 November 2013

\section{References}

1. Kolmogorov, AN: Grundbegriffe der Wahrscheinlichkeitsrechnung. Springer, Berlin (1933)

Liu, B: Uncertainty Theory, 2nd ed. Springer, Berlin (2007)

Liu, B: Why is there a need for uncertainty theory? J. Uncertain Syst. 6(1), 3-10 (2012)

Liu, B: Some research problems in uncertainty theory. J. Uncertain Syst. 3(1), 3-10 (2009)

Gao, X: Some properties of continuous uncertain measure. Int. J. Uncertain. Fuzz. 17(3), 419-426 (2009)

Zhang, ZM: Some discussions on uncertain measure. Fuzzy Optimization Decis. Making. 10(1), 31-43 (2011)

Peng, ZX, Iwamura, K: Some properties of product uncertain measure. J. Uncertain Syst. 6(4), 263-269 (2012)

. Peng, ZX, Iwamura, K: A sufficient and necessary condition of uncertainty distribution. J. Interdiscip. Math. 13(3), 277-285 (2010)

9. Liu, B: Uncertainty Theory: A Branch of Mathematics for Modeling Human Uncertainty. Springer, Berlin (2010)

10. Liu, B: Toward uncertain finance theory. J. Uncertainty Anal. Appl. 1(1) (2013)

11. Liu, YH, Ha, MH: Expected value of function of uncertain variables. J. Uncertain Syst. 4(3), 181-186 (2010)

12. Yao, K: A formula to calculate the variance of uncertain variable (2013). http://orsc.edu.cn/online/130831.pdf

doi:10.1186/2195-5468-1-9

Cite this article as: Liu: Polyrectangular theorem and independence of uncertain vectors. Journal of Uncertainty Analysis and Applications 2013 1:9. 